\title{
Das Antoniusspital in Brig
}

\author{
Von Stefan Loretan
}

Während die Entwicklung der großen Stadtspitäler schon lange in Monographien und Überblicksarbeiten dargelegt ist, wird den zahlreichen kleinen Landspitälern in der Schweiz erst in jüngerer Zeit eingehendere Beachtung geschenkt. Daß dem so ist, liegt teils wohl an den mangelhaften und zeitweise gar fehlenden Quellen, teils aber auch daran, daß viele Spitäler nicht mehr weitergeführt und andere erst im letzten und in diesem Jahrhundert gegründet worden sind.

Wohl liegt beim Briger Spital ein kontinuierliches Bestehen bis zum heutigen Tag vor; das schriftliche Quellenmaterial aber ist über viele Jahrhunderte äußerst dürftig.

Glücklicherweise sind wir wenigstens über die Gründung recht gut unterrichtet. Da auf der schon damals wichtigen Simplonroute ${ }^{1}$ zwischen den Johanniter-Hospitälern Salgesch im Mittelwallis und demjenigen auf der Paßhöhe ${ }^{2}$ arme Reisende kaum Unterkunft finden konnten, erbaten der Sittener Bürger Johann Chandeler und ein Bruder Johann von Aosta 1304 vom Bischof Bonifaz von Challant die Errichtung eines Hospitals in Brig. Im gleichen Jahr gegründet, wurde die Stiftung unter den Schutz Marias und des heiligen Antonius Eremita gestellt. Die finanziellen Mittel wurden durch Ablaßgewährung für die Wohltäter mittels einer Almosensammlung gesichert, die auch von den Bischöfen von Beley, Aosta und Genf in ihren Diözesen gefördert wurde ${ }^{3}$. Ähnliche Almosensammlungen für Hospitäler wurden damals auch von Frankreich aus ins Wallis durchgeführt ${ }^{4}$ und sind ebenfalls für das Gotthardhospiz nachgewiesen ${ }^{5}$. Schon 1360 besaß die Stiftung dann ansehnlichen Grundbesitz ${ }^{6}$.

Früh ging die Verwaltung in weltliche Hände über. Bereits 1388 erscheinen nämlich die fünf Zehnden (sie entsprechen den heutigen Bezirken des Oberwallis) als Träger des Werkes. Die erste Spitalordnung 1399 wird zwar noch vom Bischof und von den Briger Bürgern gemeinsam aufgestellt. Der anfangs durch den Bischof mit der Pfründe belehnte Spitalgeistliche wird ihm nun aber lediglich vorgestellt und muß ohne Einspracherecht bestätigt werden. Auch verfügt er nicht mehr über die Stiftungseinnahmen, sondern erhält eine feste Besoldung ${ }^{7}$. Diese Verbürgerlichung anfänglich geistlicher Stiftspitäler ist für den deutschen Raum z.B. auch in Mainz, Regensburg 
und Köln nachgewiesen. Die Briger Spitalordnung entspricht denn auch im wesentlichen dem mittelalterlichen deutschen Spitalrecht, wo der von der Stadtgemeinde gewählte Ausschuß den eigentlichen Verwalter bestimmt $^{8}$.

Neben den verwaltungstechnischen Richtlinien nehmen in dieser Ordnung vor allem Anweisungen an den Geistlichen und den Verwalter über die Gestaltung der Messe einen breiten Raum ein. Bereits 1304 und dann auch 1399 wird als Zweck des Hospitals die Ausübung der Nächstenliebe im Andenken an Christus gemäß den sechs Werken der christlichen Barmherzigkeit nach dem Matthäusevangelium angeführt. Die damalige Bedeutung des religiösen Moments geht auch aus dem Gebrauch des Sakramentes als eines eigentlichen Heilmittels hervor ${ }^{9}$. Außer der noch erhaltenen, mit 1396 datierten Spitalglocke kann auch das für die damaligen Ortsverhältnisse große Kapellenschiff in diesem Zusammenhang gesehen werden. Mit einer Flachdecke versehen und mit einem Altar an der Schmalseite könnte das saalartige Gebäude anfangs selbst als Beherbergungsraum gedient haben ${ }^{10}$. Solche Einraumanlagen als Mischform zwischen Krankensaal und Kapelle lassen sich noch heute in ganz Europa nachweisen. Als bekanntestes Beispiel möchte ich das Hôtel-Dieu in Beaune nennen.

Die erste realistische Darstellung des Briger Antoniusspitals finden wir auf einem Merian-Stich von 1643. Dort klebt ein eher schmales Gebäude an der Längsseite der Kapelle. 1494 war ein etwas drückend wirkendes spätgotisches Kreuzgewölbe ins Schiff eingezogen worden. Als Vorbild solcher Saalbauten, wie einer auch in der Leprösenkirche in Villeneuve verwirklicht worden ist, gilt das Langhaus der 1236 vollendeten Doppelkirche in Assisi ${ }^{11}$. Heute ist die Kapelle durch einen neugotischen Turm und den barocken Chor verändert. Der gotische Altar ist nicht mehr erhalten, und die Glasscheiben sind schon lange verkauft. Geblieben ist lediglich eine große spätgotische Antoniusfigur.

Bis ins frühe 17. Jahrhundert liegen dann nur spärliche Hinweise auf das Briger Spital vor. So wird 1578 die Bundeserneuerung mit den katholischen Orten in der Kapelle abgehalten. 1590 wird das «katholische christliche Exercitio oder die gute Polizeyordnung» gelobt, mit der die Bürger das Haus der Armen führen ${ }^{12}$. Der heute vor allem durch sein Schloß bekannte Große Stockalper plante einen großzügigen Neubau, der wohl durch seinen politischen Sturz verhindert wurde. Immerhin beschenkte er die Stiftung mit bedeutenden Gütern, dem barocken Hochaltar und einer Orgel. In diesem Zusammenhang sei noch erwähnt, daß Kaspar Jodok von Stockalper 1655 
den alten, schon 1235 bestehenden Jakobsspital auf dem Simplon übernahm und freiwillig auf eigene Rechnung weiterführte ${ }^{13}$.

Im 17. und 18. Jahrhundert erscheint die Spitalstiftung als wichtiger örtlicher Geldgeber mit weitgestreuten Kapitalien. Weitere Einnahmen brachte die eigene Landwirtschaft, Erbvergabungen und natürlich der Opferstock in der Kapelle. Gegen Ende des 19. Jahrhunderts wurde das Spital dann von der Einwohnergemeinde übernommen ${ }^{14}$.

Sind wir schon über die allgemeine Entwicklung der Stiftung nur lückenhaft unterrichtet, so liegt die Dokumentation über das tägliche Leben im Spital erst recht im argen. Durch den Hinweis auf die sechs Werke der christlichen Barmherzigkeit in der Ordnung von 1399 ist immerhin das breite Spektrum der dort Aufgenommenen angetönt. Wenn man die Gefahren einer damaligen $\mathrm{Paßw}$ anderung bedenkt, versteht man aber den fließenden Übergang zwischen Herberge und Krankenstube wohl etwas besser. Die fehlende Unterscheidung von Kranken, Armen und Pilgern ist für den alten Hospitaltyp noch weit übers Mittelalter hinaus charakteristisch. Da bis ins 17. Jahrhundert jegliche Erwähnung der Spitalinsassen fehlt, kann der Übergang zur vorwiegenden Krankenbetreuung für Brig zeitlich nicht umgrenzt werden. 1646 jedenfalls muß sie bereits bedeutend gewesen sein, wurde doch damals einer Wundärztin ein Viertel der Zinseinnahmen als Lohn ausbezahlt. Auch werden nun Einheimische dort gepflegt.

Im 19. Jahrhundert finden wir dann im Spital eine bunt zusammengewürfelte Gesellschaft aus «gesunden und kranken Pilgern und Reisenden, Krüppelhaften, Wahnsinnigen, Blinden und zur Arbeit Untauglichen». Daneben sind des öftern Geburten vermerkt, und unter den eingekauften Pfründnern befand sich einmal sogar eine ganze Familie. Die Fremden hatten beim Vogt um eine Eintrittskarte nachzusuchen und in der Regel damit Anrecht auf drei Mahlzeiten und ein Nachtlager. Falls die Krankheit über Gebühr lange dauerte, wurden die Bettlägrigen manchmal ins nächste Spital gefahren, von wo sie nach einigen Tagen wiederum weitergegeben wurden. 1831 wird eine Werkstatt und eine Wollspinnerei erwähnt, in denen die Insassen arbeiteten. $\mathrm{Ob}$ es sich hier um eine Frühform von Ergotherapie handelte, ist allerdings nicht ersichtlich ${ }^{15}$.

Die Kranken wurden durch einen in der Stadt niedergelassenen Arzt betreut, der die Instrumente und Medikamente selber mitbrachte. Fehlte ein Mediziner, wurden auch heilkundige Nonnen aus dem benachbarten Ursulinenkloster beigezogen. Aus den Unterlagen des 19. Jahrhunderts lassen sich an Arzneien lediglich «Seifenbaumstidel» und Amidon ausmachen. Dagegen 
werden häufig frisches Fleisch, Weißbrot, Wein und Schokolade eigens für die Kranken gekauft. Als ein Kuriosum sei noch die Vergebung eines Stipendiums an einen Medizinstudenten vermerkt ${ }^{16}$.

Einen starken Anstieg der Bettenbelegung brachte um die Jahrhundertwende der nun bis ins Oberwallis vorangetriebene Ausbau des Schienennetzes. Die verwundeten und erkrankten Arbeiter der Gornergrat- und der Simplonbahn bewirkten freilich bald eine völlige Überbelegung. Die Gesellschaft der Simplontunnelunternehmung unterhielt daher von 1899 an unter dem Betriebsarzt Dr. Daniel Pometta ein eigenes Arbeiterspital ${ }^{17}$. Mit Hilfe der Berner Alpengesellschaft, die damals die Lötschberglinie baute, konnte 1908 dann das «Oberwalliser Kreisspital» mit 80 Betten errichtet werden ${ }^{18}$. Dessen Gebäude sind nun 1978 durch einen 200 Betten aufweisenden Neubau ersetzt worden.

\section{Quellenangaben}

${ }^{1}$ Schreiber, Georg, Mittelalterliche Alpenpässe und ihre Hospitalkultur. In: Fontes Ambrosiani, Bd.27, Miscellanea G.Galbiati Bd.3, Mailand 1951, S. 350.

${ }^{2}$ Arnold, Peter, Die Johanniter im Wallis. In: 36. Heft der Ritterhausgesellschaft Bubikon, Bubikon 1973, S. 1-23.

${ }^{3}$ Joller, Franz Josef, Spital der Stadt Brig. In: Blätter aus der Walliser Geschichte, Bd.1/2, Brig 1890, S. 111-127.

${ }^{4}$ Gruber, Eugen, Die Stiftungsheiligen der Diözese Sitten im Mittelalter, Diss. Freiburg 1932, S. 123.

5 Motta, Emilio, Personaggi celebri attraverso il Gottardo. In: Bollettino storico di Svizzera italiana, Bd.4 (1884), S. 147.

${ }^{6}$ Gremaud, Jean, Documents relatifs à l'histoire du Valais, Lausanne 1875-1898, Bd.V, Nr. 182.

7 Joller, op. cit., S. 121 und 122.

8 Reike, Siegfried, Das deutsche Spital und sein Recht im Mittelalter, Bd.1, Stuttgart 1932, $\mathrm{S} .156 \mathrm{ff}$.

9 Rohde, Johann, Soziologie des Krankenhauses, Stuttgart 1962, S. 67.

${ }^{10}$ Loretan, Stefan, Das Antoniusspital in Brig. In: Oberwalliser Kreisspital Brig, Brig 1978, S. 19.

11 Gantner, Joseph, Kunstgeschichte der Schweiz, Bd.2, Frauenfeld 1947, S. $101 \mathrm{ff}$.

12 Joller, op. cit., S. 116.

13 Arnold, Peter, Der Große Stockalper, Bd. 1 und 2, Brig 1953, Bd. 1, S. 148-150, S. 156; Bd.2, S. $177 \mathrm{ff}$.

14 Loretan, op. cit., S. 17.

15 Schwäller, (unbekannt), Der wandernde Bote durch das Wallis, Hauskalender fürs Stadt- und Landvolk, Brig/Zug 1831, S.48. 
${ }^{16}$ Burgerarchiv Brig, Mappe: Spital, D 1.

17 Pometta, Daniel, Sanitäre Einrichtungen und ärztliche Erfahrungen beim Bau des Simplontunnels 1898-1906, Winterthur 1906.

${ }^{18}$ Kreisspital für das Oberwallis. Erster Jahresbericht, Brig 1909.

\section{Summary}

The Antonius hospital in Brig (Valais, Switzerland) was founded by the bishop of Sion in 1304 to carry out the six works of Christian charity on needy travellers and pilgrims crossing the Simplon pass. The funds were borne by alms and the returns of the real estate. Already in 1388 the foundation was managed by the citizens of the German speaking part of Valais.

In the beginning the chapel may have been the proper lodge-room. Up to the 19th century, the aimed population changed in favour of sick persons. They were attended by established medical practitioners and nursed by a male-servant. Medicine was frequently furnished by the nearby Ursuline convent.

In 1908 the old hospital was replaced by the "Hospital of the Upper Valais".

Stefan Loretan

Termerweg 21

CH-3900 Brig 